\title{
Design, Effektgrösse und Power von Ayurveda-Studien zu Typ 2 Diabetes mellitus
}

\author{
Christian H.S. Kessler, Thorsten Doering \\ Deutsche Klinik für Integrative Medizin und Naturheilverfahren, Bad Elster, Deutschland
}

$D^{2}$ ie ayurvedische Medizin wird in Südasien seit über 2000 Jahren auf breiter Basis als Volksmedizin praktiziert und ist damit eines der ältesten Gesundheitssysteme der Welt. Ayurveda wird von der Weltgesundheitsorganisation (WHO) als medizinische Wissenschaft anerkannt. Allein in Indien sind laut WHO mehr als 300.000 ayurvedische Ärzte registriert, an über 200 von der indischen Regierung akkreditierten Universitäten und Colleges wird Ayurveda-Medizin systematisch gelehrt und angewandt. So beträgt beispielsweise die universitäre Regelstudienzeit für den Bachelor of Ayurvedic Medicine and Surgery 5 1/2 Jahre [1,2].

\section{Ayurveda - die traditionelle indische Medizin}

Aus der Sanskrit-Sprache übersetzt bedeutet Ayurveda „Wissen[-schaft] vom Leben“. Die Philosophie des Ayurveda hat im Laufe der Jahrtausende komplexe medizinische Theorien hervorgebracht. Sie postuliert eine grundsätzliche Harmonie zwischen dem Mikrokosmos Mensch und dem Makrokosmos Umwelt. Ayurveda ist nicht nur ein medizinisches System, sondern zugleich eine Lebensphilosophie und findet sowohl in Therapie als auch Prävention breite Anwendung [2,3,4,5,6].

Ayurveda spielt mittlerweile auch in Europa eine zunehmende Rolle. Vor allem in Deutschland gehört die ayurvedische Medizin zu den am schnellsten wachsenden CAM (Complementary and Alternative Medicine)-Verfahren. Einige Aspekte von Ayurveda, zum Beispiel Öl-Massagen, Konstitutionstypenbestimmung und ayurvedische Ernährungslehre, sind bereits schon jetzt, vor allem
Hintergrund: Diabetes mellitus ist in der ayurvedischen Medizin bereits seit über 2000 Jahren bekannt und wird in den systemeigenen Lehrwerken übereinstimmend als Madhumeha (süsser Durchfluss) bezeichnet. Angenommen wird eine multifaktorielle Krankheitsentstehung. Primäres Therapieziel ist die Korrektur der aus dem Gleichgewicht geratenen Funktionsprinzipien (Doshas). Es existieren auffällige Parallelen zur westlichen Vorstellung des Diabetes mellitus. Zielsetzung: Ausgangspunkt ist die systematische und umfassende Suche nach der bestmöglichen Evidenz zu ayurvedischen Therapieverfahren des Typ-2-Diabetes. Auf der Basis des Designs, der Stärke der Effekte und der Präzision der Schätzungen sollten Aussagen zu Wirksamkeit und möglichem Nutzen ayurvedischer Diabetes-Interventionen gemacht werden. Methodik: Basierend auf zwei systematischen Reviews zu ayurvedischen Therapiemethoden bei Diabetes mellitus wurden anhand vordefinierter Auswahlkriterien 25 Ayurveda-Studien zu Effektivität und Power untersucht. Die Beurteilung der Studien wurde systematisiert, indem vier wesentliche Aspekte nacheinander bearbeitet wurden: Design, Gültigkeit (interne Validität), Grösse und Präzision sowie Übertragbarkeit und Anwendbarkeit der Ergebnisse. Wegen der stark unterschiedlichen Aussagekraft wurden die Ergebnisse der kontrollierten und nicht-kontrollierten Studien separat dargestellt. Ergebnisse: 7 kontrollierte und 18 nicht-kontrollierte Studien erfüllten die Ein- und Ausschlusskriterien: Die Differenz der Mittelwerte von Nüchternglukose lag im Phytotherapiearm im Durchschnitt bei einer Senkung um 42mg/dl, im Vergleichsarm um 13mg/dl. Die Differenz der Mittelwerte des HbA1c wurde im Phytotherapiearm um durchschnittlich 2,8\% gesenkt, im Vergleichsarm um 0,3\%. Der Phytotherapiearm war im Mittelwert um 70\% besser als der Vergleichsarm. Die Effektstärke war in $75 \%$ der Studien gross und in $17 \%$ gering. Die Power war bei $66 \%$ der Studien sogar besser als 0,9. Für einige Pflanzen, wie beispielsweise Coccinia indica, Gymnema sylvestre und Trigonella foenum graecum existiert bereits eine Reihe randomisierter Studien, die eine erste EBM (Evidence Based Medicine)-Eingruppierung erlauben. Schlussfolgerungen: Ayurvedische Diabetes-Studien zeigen, dass die Effektgrösse der Phytotherapien in der Mehrzahl gross ist. Bei mehr als 45 verschiedenen Behandlungsformen des Typ-2-Diabetes ist jedoch die Anzahl gut geplanter RCTs (Randomized Controlled Trials) pro Verfahren für eine abschliessende Bewertung nach EBM noch zu gering. Durch an Ayurveda angepasste Phase III-Studien mit mulitzentrischen/ multinationalen Studiendesigns und externer Bewertung könnte das Evidenzniveau erhöht werden.

Schlüsselwörter: Diabetes mellitus, Ayurveda, Effektstärke, Poweranalyse

\section{Design, Effect Size and Power of Ayurvedic Studies on Type 2 Diabetes}

Background: In Ayurveda, diabetes has been well-known for over 2000 years and is universally known as Madhumeha (sweet flow) in Ayurvedic medical textbooks. A multifactorial pathogenesis is assumed and therapy aims primarily at adjusting imbalance in the body's primary life forces (doshas). There are noticeable parallels to western medicine's view of diabetes. Aim of the Study: The initial goal was to conduct a systematic and comprehensive search for the best evidence of Ayurvedic therapies of type 2 diabetes. Then, based on study design, size of effects and precision of estimates, the level of evidence and potential benefits, the selected Ayurvedic diabetes interventions would be investigated. Methods: From two systematic reviews of Ayurvedic therapies of diabetes, 25 Ayurvedic studies were selected by predefined criteria and assayed on effectiveness and power. The assessment of the studies was systematized by handling four major issues: Validity (internal validity), size and precision as well as transferability and applicability of the results. Because of the widely varying significance, results of controlled and non-controlled studies were described separately. Results: 7 controlled and 18 non-controlled studies met the inclusion and exclusion criteria: Mean-values (pre- and postinterventional) of fastingblood-sugar showed an average fall of $42 \mathrm{mg} / \mathrm{dl}$ in the phythotherapy arm versus $13 \mathrm{mg} / \mathrm{dl}$ in the comparison arm. The mean difference of $\mathrm{HbA} 1 \mathrm{c}$ fell $2.8 \%$ versus $0.3 \%$. Mean-values of the phytotherapy arms were $70 \%$ better than in comparison arms. The effect size in $75 \%$ of the studies was large, and small in $17 \%$. The power was larger than 0.9 in $66 \%$ of the analyzed studies. For some plants used in Ayurveda, e.g. Coccinia indica, Gymnema sylvestre and Trigonella foenum graecum, a notable number of randomized controlled trials (RCTs) exist which allow a first Evidence Based Medicine (EBM) classification. Conclusions: The majority of the 7 controlled and 18 non-controlled studies showed that the effect sizes of the phytotherapy arms are large. Yet for more than 45 different forms of treatment of type 2 diabetes, the number of well-planned RCTs per procedure is still too small for a final evaluation according to EBM. Well-designed, peerreviewed phase III studies adapted to Ayurveda are required to support and enhance the level of evidence.

Key words: Diabetes mellitus, Ayurveda, effect size, power analysis 
in der Wellness-Bewegung, feste Bestandteile von Angebot und Nachfrage in der CAM-Szene. Die wachsende Anzahl von Kliniken mit Ayurveda-Angeboten in Deutschland und die zunehmende Berücksichtigung von Ayurveda bei der Erstellung universitärer Lehrmaterialen weisen auf eine steigende Bedeutung der ayurvedischen Medizin hin. Ein Blick in medizinische Datenbanken, wie zum Beispiel die PubMedDatenbank der National Library of Medicine, macht zudem deutlich, dass während der letzten Jahre die Forschungsaktivitäten in diesem Bereich stark zunehmen. Allein mit dem Suchbegriff

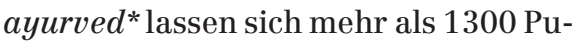
blikationen zu Ayurveda isolieren [9].

Die konstitutionsbasierte Theorie der 3 Funktionsprinzipien Vata, Pitta, Kapha (Tridosha-Theorie) bildet die philosophische Grundlage für das ayurvedische Medizinsystem. Das Zusammenwirken der drei Funktionsprinzipien bestimmt und regelt demnach alle Vorgänge im Organismus. Sie stehen in einem empfindlichen und dynamischen Gleichgewicht zueinander und sind voneinander abhängig. Dies sind Vata (kinetisches Prinzip), Pitta (metabolisches Prinzip), Kapha (anaboles Prinzip). Sie prägen von Geburt an individuelle Körpermerkmale und die Grundlagen der Persönlichkeitsstruktur eines Menschen. Die auf diesem Dogma basierende Konstitutionslehre (Prakrti) beschreibt die individuelle Konstitution eines Menschen, die zusätzlich durch äusserliche Faktoren wie Ernährung, Verhalten, soziales Umfeld, Klima, Emotionen und individuelle Verhaltensweisen beeinflusst wird. Geraten die individuell unterschiedlich austarierten Funktionsprinzipien aus ihrem Equilibrium, kommt es zur Entstehung von Krankheiten [3,4,6,7,8].

Im Gegensatz zu den hoch differenzierten diagnostischen und therapeutischen Ansätzen sowie den vielfältigen Angeboten für Ayurveda steckt die wissenschaftliche Aufarbeitung gemäss den Grundsätzen der EBM noch in den Anfängen. Für Ärzte und Patienten ist es deshalb oft schwierig, Therapieentscheidungen auf der Basis der besten verfügbaren Evidenzen $\mathrm{zu}$ treffen. Trotz Studien zu verschiedenen ayurvedischen Therapiemodalitäten fehlen in vielen Bereichen nach wie vor Antworten auf Kernfragen zu Wirkweise und Wirksamkeit ayurvedischer Therapienmethoden oder sind mittels konventioneller Retrieval-Strategien nicht leicht zu finden. Diese Schwachstelle gilt es zu beseitigen. Auch ein traditionelles Medizinsystem wie Ayurveda, das sein Wissen während der letzten 2000 Jahre vor allem aus Erfahrungswerten generiert und legitimiert hat, muss sich weiterentwickeln.

Anstelle des bisher unbefriedigenden Austauschs zwischen Schul- und Komplementärmedizin sollte ein rationaler Diskurs entstehen, mit dem Ziel einer patientengerechten Integration verschiedener therapeutischer Schulen ( $\rightarrow$ medizinischer Pluralismus) [53]. Bereits seit dem Zweiten Arzneimittelgesetz ist der Pluralismus der Therapieansätze auch mit der Frage nach dem Pluralismus der Evaluationsmethoden verbunden: Sind für Ayurveda eigene, spezifische Theorien oder Methoden der Evaluation (speziell zur Wirksamkeits- oder Nutzenevaluation) vorhanden, sinnvoll, erforderlich? Gibt es besondere Bedingungen, die für Ayurveda oder für Teile des Ayurveda andere Anforderungen an Evaluation erforderlich machen als in der Schulmedizin?

Um zukünftig einen Pluralismus der Therapieansätze zu akzeptieren, müssen von Schulmedizin und CAM gleichermassen ideologische Barrieren überwunden werden. Am Beispiel von Ayurveda gilt hierbei zu klären, wo die ayurvedische Medizin ihre Stärken hat und als eine sinnvolle Ergänzung der Schulmedizin eingesetzt werden könnte. Versorgungsforschung und HTA (Health Technology Assessment) könnten hier wesentliche Beiträge leisten. Wenn es in der Zukunft gelingt, entsprechende Handlungsfelder zu identifizieren, lägen gerade dort sinnvolle Anwendungsbereiche für (evidenzbasierte) ayurvedische Therapieansätze.

Solche Handlungsfelder sind z.B. chronische Erkrankungen wie z.B. Typ-2-Diabetes mellitus. Hier ist die Schulmedizin, trotz aller Fortschritte, noch schwach.

Weltweit sind zur Zeit rund 5,1\% aller Menschen an Diabetes mellitus erkrankt. Der Diabetes mellitus liegt an vierter Stelle der Haupttodesursachen in den Industrieländern. Mit rund sechs Millionen Betroffenen liegt die Quote in Deutschland bei circa sieben Prozent $[49,50]$. Die Deutsche Diabetes-Union schätzt die Dunkelziffer noch wesentlich höher (Deutscher Gesundheitsbericht Diabetes 2003). Eine weitere Zunahme ist zu erwarten. Eine Hochrechnung geht davon aus, dass sich die Anzahl der Diabetes-Kranken in Deutschland bis 2020 von heute ca. 6,3 Millionen auf voraussichtlich 10-11 Millionen fast verdoppeln wird. Die Kosten für die Therapie des Typ-2-Diabetes mellitus und seiner assoziierten Erkrankungen in Deutschland betrugen 1998 bereits mehr als 16,1 Milliarden Euro jährlich [49,50]. Allein die gesetzlichen Krankenversicherungen gaben 9,5 Milliarden Euro für die Behandlung dieser Patienten aus. 50 Prozent der Krankenversicherungskosten entfielen auf die stationäre und 13 Prozent auf die ambulante Therapie. Die Kosten für direkte Diabetes-Medikation (Insulin, orale Antidiabetika) machen gerade sieben Prozent der Gesamtkosten aus. Sobald ein Patient an den Spätfolgen des Diabetes erkrankte, vervierfachten sich die Ausgaben im Vergleich zu einem durchschnittlichen gesetzlich Krankenversicherten.

Ausgangspunkt dieser Arbeit ist die systematische und umfassende Suche nach der bestmöglichen Evidenz für ayurvedische Therapieverfahren des Typ-2-Diabetes. Die Beurteilung der Studien wurde systematisiert, indem 4 wesentliche Aspekte nacheinander bearbeitet wurden: Design, Gültigkeit (interne Validität), Grösse und Präzision sowie Übertragbarkeit und Anwendbarkeit der Ergebnisse. Im Rahmen dieser Aspekte wurden Outcomeund Poweranalysen durchgeführt, um folgende Fragestellungen $\mathrm{zu}$ beantworten:

- Lässt sich Ayurveda am Beispiel Typ-2-Diabetes mellitus nach den Prinzipien von Cochrane untersuchen?

- Was ist die beste verfügbare externe Evidenz für Typ-2-Diabetes mellitus?

- Wie gross sind Effektivität und Power der eingeschlossenen Studien?

- Kann die Outcome-Qualität entsprechender Ayurveda-Therapien mit der 
Outcome-Qualität schulmedizinischer Therapien verglichen werden? Was sind die möglichen Unterschiede

- Kann die Integration bestimmter ayurvedischer Diabetestherapien, dort wo dies sinnvoll erscheint und Wirksamkeiten in wissenschaftlichen Studien nachgewiesen werden konnten, einen Beitrag zu einer effektiveren, effizienteren und letztlich auch kostengünstigeren Gesundheitsversorgung leisten?

\section{Ayurveda und Diabetes mellitus}

Der Diabetes mellitus ist in der ayurvedischen Medizin bereits seit über 2000 Jahren bekannt und wird in den systemeigenen Lehrwerken übereinstimmend als Madhumeha (süsser Durchfluss) bezeichnet. Angenommen wird eine multifaktorielle Krankheitsentstehung. Äquivalentvorstellungen genetischer Prädispositionen spielen in der ätiopathogenetischen Deutung eine genauso bedeutsame Rolle wie die Annahme davon unabhängiger erworbener Faktoren. Im Zentrum steht das Postulat eines individuellen Ungleichgewichtes der drei Funktionsprinzipien, v.a. von Vata und Kapha. Vorbestehende Schwächen der Körpergewebe (Dhatus), vor allem von Meda (Fettgewebe), Mamsa (Muskelgewebe) und Vasa (Muskelfettgewebe) tragen zur Manifestation des Diabetes mellitus bei. Dennoch existieren auffällige Parallelen zur westlichen Vorstellung des Diabetes mellitus: Beispielsweise unterscheidet auch Ayurveda zwischen einer „genetisch“ bedingten Form der Erkrankung (Sahaja Madhumeha), die mit dem Typ-1-Diabetes korreliert werden kann, und einer „erworbenen“ Form (Apathiyanimittaya Madhumeha), die dem Typ-2-Diabetes mellitus entspricht (Abb. 1) [12,13,14,15,16,17].

Primäres Therapieziel ist die Korrektur der aus dem Gleichgewicht geratenen Funktionsprinzipien (Doshas), hier insbesondere von Kapha und Vata.

Interindividuelle Unterschiede in der ayurvedischen Therapiestrategie beim Diabetes mellitus ergeben sich aus dem körperlichen Zustand der Patienten: Der adipöse Typ-2-Diabetiker

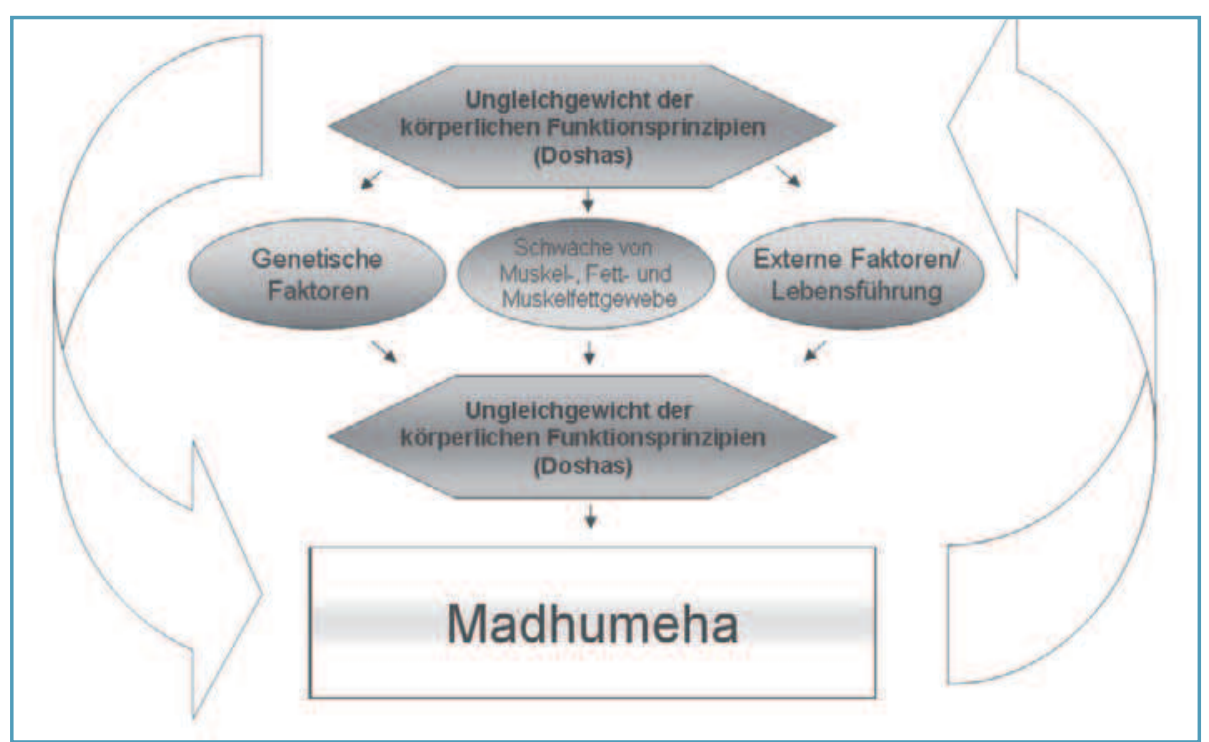

Abb. 1. Ayurvedische Ätiopathogenese des Diabetes mellitus (Madhumeha).

wird zu Beginn der Therapie einer ausführlichen Reinigungsprozedur unterzogen (Shodhana). Bei ausgezehrten, sich in einem schlechten Allgemeinzustand befindlichen Typ-2-Diabetikern wird in der Regel zuerst auf sanftere Methoden ausgewichen. Auf diese Vorbehandlungen (Purvakarma) folgt ein Therapieschema, welches sich schwerpunktmässig auf Phytotherapie, Ernährungstherapie und körperliche Aktivität stützt. Die Therapie ist stets individuell ausgerichtet und üblicherweise multimodal, im modernen Ayurveda steht jedoch die Phytotherapie im Vordergrund (Abb. 2) [12,13,14,15,16,17].

\section{Methoden}

Basierend auf zwei systematischen Reviews zu ayurvedischen Therapiemethoden bei Diabetes mellitus [18,19] wurden anhand vordefinierter Auswahlkriterien Ayurveda-Studien zu Effektivität und Power untersucht. Studien, welche die folgenden Kriterien erfüllen konnten, wurden miteinbezogen:

- RCTs jeder Studiengrösse.

- CCTs mit mindestens einem Studienarm mit $\geq 10$ Studienteilnehmern.

- Studien niedrigerer Evidenzkategorien (zum Beispiel Fall-Kontroll-Studien oder Kohortenstudien) mit $\geq 10$ Studienteilnehmern pro Studienarm. - Der/die im Rahmen der Studie un- tersuchte/n Wirkstoff/e musste/n dem Studienteilnehmer mehr als $1 \mathrm{x}$ verabreicht werden.

- Die Studien mussten mindestens einen der folgenden Zielparameter beinhalten:

- HbA1c

- Nüchternblutglukose

- postprandiale Glukose.

- Aufführung der Anzahl der Studienteilnehmer, Prä- und Postinterventionswerte und Standardabweichungen / Standardfehler für jeden Studienarm.

\section{Effektgrössenberechnung}

Die Effektstärke (ES) mit ihrem 95\%Konfidenzintervall erlaubt es, die Frage der „praktischen Bedeutsamkeit“ eines Effektes abzuschätzen. Es gilt:

- Je grösser der Unterschied zwischen den experimentellen Bedingungen, desto grösser die Effektstärke.

- Je kleiner die Varianz innerhalb der Bedingungen, desto grösser die Effektstärke.

Zur Berechnung der Effektstärke nach Cohen werden zuerst anhand von Mittelwerten, Standardabweichungen und Gruppengrössen die Rohdifferenz und die „gepoolte“ Standardabweichung berechnet. Die standardisierte Effektstärke nach Cohen errechnet sich dann als Quotient dieser beiden Grössen, die nach HEDGES [52] noch biaskorrigiert wird. 


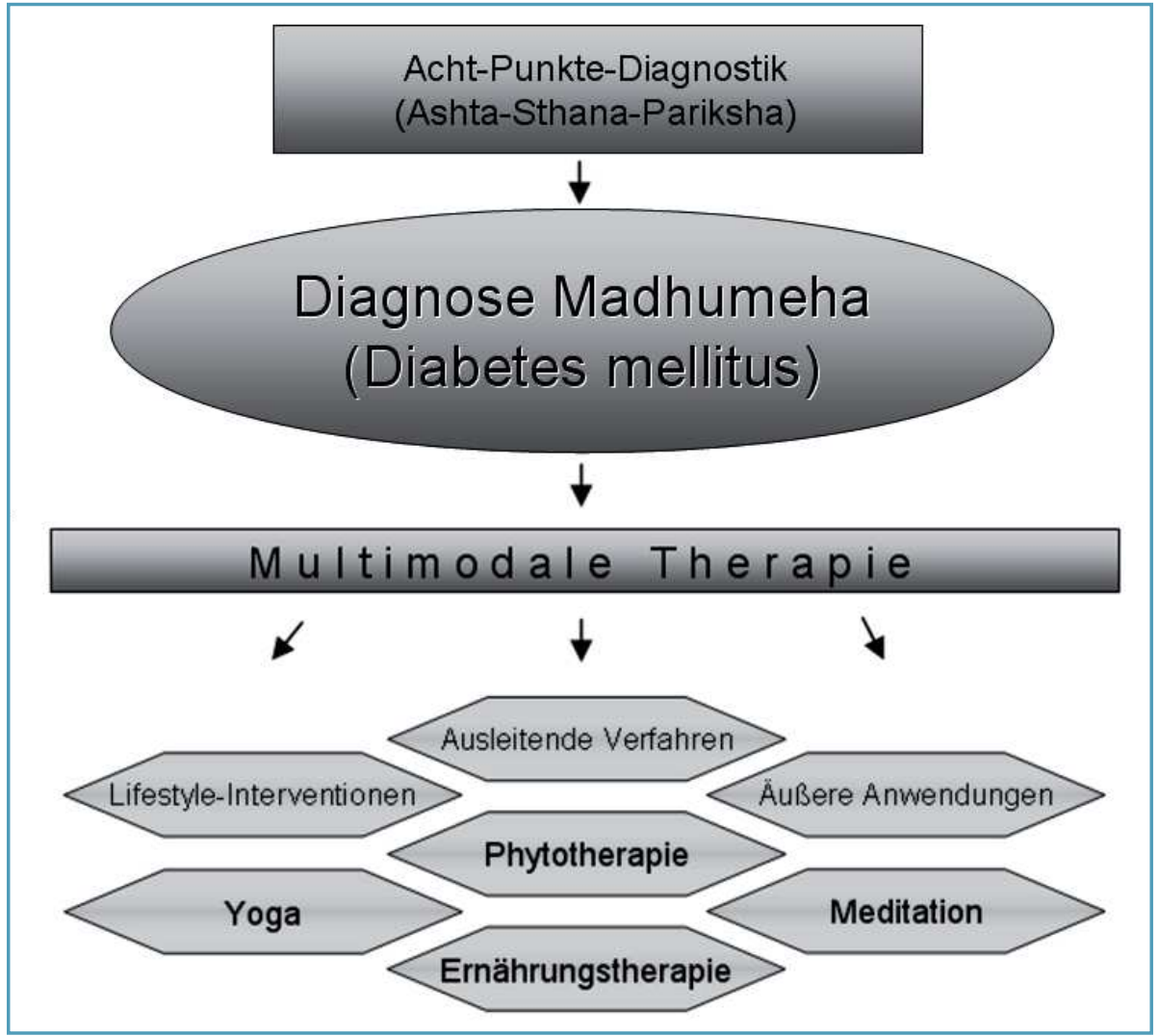

Abb. 2. Diagnose und Therapie von Madhumeha (Diabetes mellitus).

Es gibt keine allgemeingültigen Regeln für die Interpretation von Effektgrössen, jedoch existiert eine Forschungstradition, deren Analyse bei der Interpretation helfen kann. So hat CoHEN [51] folgende Konventionen für die Interpretation der Effekte eingeführt: Kleiner Effekt $(0,2 \leq \mathrm{ES}<0,5)$, mittlerer Effekt $(0,5 \leq \mathrm{ES}<0,8)$, grosser Effekt $(E S \geq 0,8)$. Berechnet werden: die Rohdifferenz, die "gepoolte" Standardabweichung, der p-Wert, die mittlere Differenz, das 95\% CI für die Differenz, die Effektstärke, die biaskorrigierte Effektstärke nach HEDGES, SEM der ES und das Konfidenzintervall für die Effektstärke. Im Falle von RCTs und CCTs vergleichen wir den Phytoarm mit dem Kontrollarm. Hierzu berechnen wir die Differenz der Differenzen, d.h. die Präwerte des Phytoarms minus die Postwerte des Phytoarms minus [die Präwerte des Kontrollarms minus die Postwerte des Kontrollarms]. Die übrigen Berechnungen wie 95\% CI für die Differenz, die Effektstärke, die biaskorrigierte Effektstärke nach HEDGES werden in Analogie für den Fall der Differenz von abhängigen Mittelwerten durchgeführt [51,52].

\section{Poweranalysen}

Unter Power (=statistische Trennschärfe) versteht man die Fähigkeit einer Studie, einen tatsächlich vorhandenen Unterschied statistisch signifikant nachzuweisen und die Nullhypothese zu verwerfen, wenn sie tatsächlich falsch ist. Der Nachweis bezieht sich auf a priori festgelegte Unterschiede in den Endpunkten von Therapie- und Kontrollgruppe.

Neben den verwendeten statistischen Tests und dem tatsächlichen Unterschied zwischen den Studienarmen hängt die Power einer Studie noch von der Variabilität (hier Standardabweichung) und vom Stichprobenumfang ab. Praktisch wird man eine Phytotherapie annehmen, wenn eine deutliche Symptomverbesserung (Effektstärke $\geq$ $0,5)$ mit einer Irrtumswahrscheinlichkeit von $5 \%$ nachgewiesen wird. Die Power einer Studie sinkt mit wachsender Streuung, steigt mit zunehmendem Stichprobenumfang und steigt mit grösser werdender Differenz $\mu_{1}$ und $\mu_{2}$. Das heisst, grosse Gruppenunter- schiede bei geringer Variabilität können mit einer vergleichsweise kleinen Fallzahl statistisch entdeckt werden, kleine Unterschiede bei hoher Variabilität erfordern dagegen grosse Fallzahlen. Es wird also deutlich, dass zu einer guten Planung von klinischen Studien und letztendlich auch zu einer guten Präsentation der Ergebnisse insbesondere die Spezifizierung von

- den zu entdeckenden Unterschieden,

- der zu erwartenden Variabilität und

- der gewünschten Power mit der daraus resultierenden Fallzahl gehören - sowie die statistischen Verfahren, die zur Analyse der Daten herangezogen werden sollen oder wurden.

\section{Ergebnisse}

\section{Studienlage}

Vor Durchführung der Signifikanztests, der Berechnung der Effektgrössen sowie von retrospektiven Poweranalysen konnten - basierend auf den vorhandenen Evidenzen - folgende generelle Beobachtungen gemacht werden:

- Der Typ-2-Diabetes mellitus ist das Krankheitsbild, für das am häufigsten Studien zu ayurvedischen Therapie-Interventionen durchgeführt wurden [18].

- $90 \%$ der Studien lassen sich in die Evidenzklassen IIa, IIb und III einordnen.

- Es existiert eine Anzahl an RCTs $(10 \%, n=7)$. Jedoch ist die methodologische Qualität (bewertet u.a. mit dem JADAD-Score [20]) in der Mehrzahl der Fälle moderat bis mangelhaft.

- Die Anzahl von Studien aus der höchsten Evidenzkategorie (z.B. Systematische Reviews, Metaanalysen) ist klein. Es existieren lediglich zwei Studien der Evidenzkategorie Ia für Diabetes mellitus $[18,19]$

- $>90 \%$ der vorgenommen Interventionen stammen aus dem Bereich der Phytotherapie.

n Die beste Evidenz liegt für die Heilpflanzen Coccinia indica, Gymnema sylvestre und Trigonella foenum graecum vor.

- $\mathrm{Zu}$ anderen ayurvedischen Therapiemodalitäten bei der Behandlung des Diabetes mellitus, wie Ernährung 
und ausleitende Verfahren (Panchakarma), existieren nur wenige Studien.

- Die Erfassung der Basisparameter wurde im Rahmen vieler der vorliegenden Studien nicht systematisch eingehalten. Langzeitparameter wie HbA1c - wurden nur in wenigen Studien untersucht.

- Die Mindestdauer zur Bestimmung klinischer Effekte wurde nicht immer eingehalten. Eine Studie, die den Kontrollparameter HbA1c benutzt, verlangt beispielsweise eine Mindestdauer von drei Monaten.

\section{Signifikanztests und Poweranalysen}

Da in den meisten der hier untersuchten Studien Signifikanztests fehlten oder nur ansatzweise durchgeführt wurden, haben wir alle Studien einheitlich untersucht und hierzu Signifikanztests, die Berechnung der Effektgrössen nach Cohen sowie entspre- chende Poweranalysen durchgeführt. Hierbei wurden folgende Parameter untersucht:

- Nüchternglukose,

- postprandiale Glukose und

- HbA1c.

- Es wurden 25 Studien untersucht, die den Zielparameter Nüchternglukose massen (2 RCTs, 5 CCTs, 18 Studien anderer Studiendesigns) [21 $-41]$,

- 21 Studien, die den Zielparameter postprandiale Glukose massen (2 RCTs, 1 CCT, 18 Studien anderer Studiendesigns [21,22,24,29-41] sowie

- 7 Studien, die den Zielparameter HbA1c massen (2 CCTs sowie 5 Studien anderer Studiendesigns [26,27, $28,34,40]$.

Folgende Ergebnisse konnten beobachtet werden:

- Beide zum Zielparameter Nüchternglukose untersuchte RCTs haben eine grosse Effektstärke (Bias korri- giert: 0,8 bzw. 1,3) und eine Power von 0,6 bzw. 0,97. Von den insgesamt 5 CCTs haben 2 eine grosse ES (Bias korrigiert: 1,8 und 2,2), 1 eine mittlere ES $(0,7)$ und 2 eine geringe ES $(0,2)$. Von 18 analysierten Studien anderer Studiendesigns weisen insgesamt 13 eine grosse ES, eine eine mittlere ES und die restlichen 4 Studien eine geringe ES auf (Tab. 1 und 2).

- Von den 2 RCTs mit Zielparameter postprandiale Glukose hat eine Studie eine grosse ES $(1,0)$ und eine Studie eine mittlere ES $(0,6)$. Die Power der Studie mit mittlerer ES ist zu niedrig (Power $=0,55$ ). Die einzige CCT hat ebenfalls eine hohe ES (Bias korrigiert: 1,0). Von weiteren 18 Studien weisen 15 eine grosse und 2 eine geringe ES auf. Eine Studie war nicht weiter auswertbar, da die Standardabweichungen der Präund Postmittelwerte nicht angegeben waren (Tab. 3 und 4).

Tab. 1. (Randomisierte) kontrollierte Studien: Studiengrösse, Mittelwerte vor und nach der Behandlung, Standardabweichungen, Vergleich Phytoarm mit Kontrollarm (unverbundener T-Test), Effektgrösse und Power: Nüchternblutzucker (mg/dl)

\begin{tabular}{|c|c|c|c|c|c|c|c|c|c|c|c|c|c|c|c|c|c|c|c|}
\hline \multirow{3}{*}{ Studie } & \multicolumn{7}{|c|}{ BASISDATEN } & \multicolumn{5}{|c|}{$\begin{array}{c}\text { DIFFERENZ DER } \\
\text { DIFFERENZEN }\end{array}$} & \multicolumn{6}{|c|}{$\begin{array}{c}\text { STANDARDISIERTE } \\
\text { EFFEKTGRÖSSE }\end{array}$} & \multirow[b]{2}{*}{ 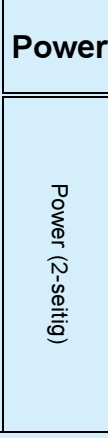 } \\
\hline & \multicolumn{4}{|c|}{ Phytoarm } & \multicolumn{3}{|c|}{ Kontrollarm } & \multirow[t]{2}{*}{ 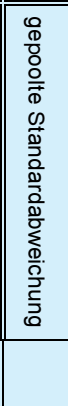 } & \multirow[t]{2}{*}{ 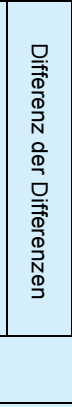 } & \multirow[t]{2}{*}{ 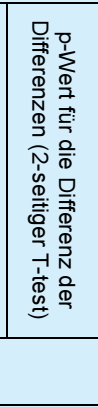 } & \multicolumn{2}{|c|}{$\begin{array}{c}95 \% \\
\text { Konfidenzinter- } \\
\text { vall für die } \\
\text { Differenz der } \\
\text { Differenzen }\end{array}$} & \multirow[t]{2}{*}{ 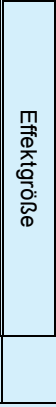 } & \multirow[t]{2}{*}{ 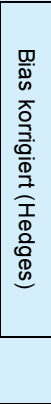 } & \multirow[t]{2}{*}{ 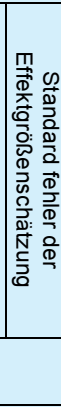 } & \multicolumn{2}{|c|}{$\begin{array}{c}\text { Konfidenzinter- } \\
\text { vall für die } \\
\text { Effektgröße }\end{array}$} & \multirow[t]{2}{*}{ 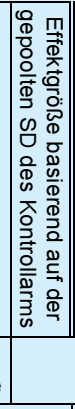 } & \\
\hline & & Mittelwert & $\mathrm{n}$ & SD & Mittelwert & $n$ & SD & & & & $\begin{array}{c}\text { untere } \\
\text { Grenze }\end{array}$ & \begin{tabular}{|c|} 
obere \\
Grenze
\end{tabular} & & & & \begin{tabular}{|c|} 
untere \\
Grenze
\end{tabular} & $\begin{array}{l}\text { obere } \\
\text { Grenze }\end{array}$ & & \\
\hline \multicolumn{20}{|l|}{$\begin{array}{l}\text { Randomisierte } \\
\text { Kontrollierte Studien }\end{array}$} \\
\hline \multirow{2}{*}{$\begin{array}{l}\text { Azad Khan } 1979 \text { / Coccinia } \\
\text { indica }\end{array}$} & Prä & 178.8 & 16 & 50.6 & 195.4 & 16 & 51.9 & \multirow{2}{*}{53.6} & \multirow{2}{*}{42.6} & \multirow{2}{*}{0.0322} & \multirow{2}{*}{3.9} & \multirow{2}{*}{81.3} & \multirow{2}{*}{ | 0.8} & \multirow{2}{*}{0.8} & \multirow{2}{*}{0.4} & \multirow{2}{*}{0.1} & \multirow{2}{*}{1.5} & \multirow{2}{*}{1.1} & \multirow{2}{*}{0.59} \\
\hline & Post & 122.1 & 16 & 46.5 & 181.3 & 16 & 50.7 & & & & & & & & & & & & \\
\hline Agrawal 1996 / Ocimum & Prä & 134.5 & 20 & 23.4 & 132.4 & 20 & 22 & 190 & 256 & | & 134 & 378 & 1.3 & 13 & 03 & 06 & 20 & 14 & 097 \\
\hline sanctum & Post & 99.7 & 20 & 17.6 & 123.2 & 20 & 18.5 & 10.0 & 25.0 & 0.0001 & 13.4 & (31.0 & 1.0 & 1.5 & 0.3 & 0.0 & 2.0 & 1.4 & - \\
\hline $\begin{array}{l}\text { Kontrollierte Klinische } \\
\text { Studien }\end{array}$ & & & & & & & & & & & & & & & & & & & \\
\hline & Prä & 153.4 & 32 & 46.7 & 156.4 & 8 & 43.6 & 27.2 & 48.4 & 0.0001 & 26.6 & 70.2 & 1.8 & 1.7 & 0.4 & 0.9 & 2.6 & 1.4 & 0.99 \\
\hline 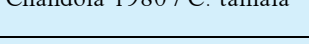 & Post & 112.7 & 32 & 41.9 & 164.1 & 8 & 61.1 & 0121.2 & 40.4 & 0.0001 & 20.0 & 70.2 & 100 & 1.1 & 0.4 & 0.3 & 2.0 & 1.4 & 0.35 \\
\hline Kamble 1996 / Coccinia & Prä & 160 & 25 & 12.1 & 165 & 15 & 20.6 & 1 & 30 & 0.4 & -58 & 118 & 0.2 & 02 & 03 & -0.4 & 09 & 02 & 009 \\
\hline indica & Post & 118 & 25 & 11.1 & 120 & 15 & 16.3 & 13.5 & 3.0 & 0.4950 & -5.8 & 11.8 & 0.2 & 0.2 & 0.5 & -0.4 & 0.9 & 0.2 & 0.09 \\
\hline Kohli 1993 / Eugenia & Prä & 163 & 28 & 81.3 & 155.7 & 6 & 73.6 & 486 & 77 & 07272 & -369 & 523 & 0.2 & 02 & 05 & -07 & 10 & 01 & 007 \\
\hline jambolana & Post & 129.6 & 28 & 63.6 & 130 & 6 & 66.5 & 40.0 & & & & & & & & & & & \\
\hline Baskaran 1990 / Gymnema & |Prä & 174.5 & 22 & 32.2 & 150 & 25 & 20.4 & 16.3 & 36.0 & 0.0000 & 26.4 & 45.6 & $\mid 2.2$ & 2.2 & 0.4 & 1.5 & 2.9 & 2.9 & 1 \\
\hline Sylvestre & Post & 145.7 & 22 & 26.6 & 157.2 & 25 & 21.1 & & & & & & & & & & & & \\
\hline Shanmugasundaram 1990 / & Prä & 231.9 & 27 & 64 & 233.1 & 37 & 45.4 & 648 & 59 & 000 & 3 & 787 & 7 & 7 & 3 & 2 & 12 & 09 & 077 \\
\hline Gymnema Sylvestre & Post & 177.1 & 27 & 75.2 & 224.2 & 37 & 1.7 & (I.0 & Th. & & 10.1 & & & 0.1 & 0.0 & & 1.2 & & \\
\hline
\end{tabular}


Tab. 2. Einzelkräuter und Kräutermischungen: Studiengrösse, Mittelwerte vor und nach der Behandlung, Standardabweichungen, verbundener T-Test, Effektgrösse und Power: Nüchternblutzucker (mg/dl)

\begin{tabular}{|c|c|c|c|c|c|c|c|c|c|c|c|c|c|c|c|c|c|c|}
\hline \multirow{3}{*}{ Studie } & \multicolumn{6}{|c|}{ BASISDATEN } & \multicolumn{5}{|c|}{ DIFFERENZ } & \multicolumn{6}{|c|}{$\begin{array}{l}\text { STANDARDISIERTE } \\
\text { EFFEKTGRÖSSE }\end{array}$} & \multirow{3}{*}{ 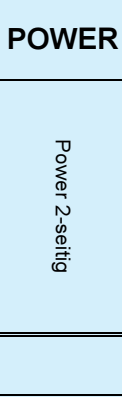 } \\
\hline & \multicolumn{3}{|c|}{ Prä } & \multicolumn{3}{|c|}{ Post } & \multirow{2}{*}{ 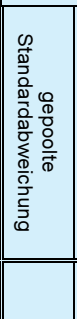 } & \multirow{2}{*}{ 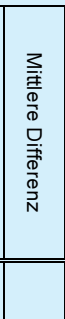 } & \multirow{2}{*}{ 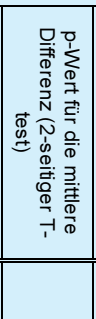 } & \multicolumn{2}{|c|}{$\begin{array}{c}95 \% \\
\begin{array}{c}\text { Konfidenzintervall für } \\
\text { die Differenz }\end{array}\end{array}$} & \multirow[t]{2}{*}{ 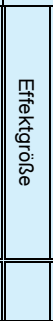 } & \multirow{2}{*}{ 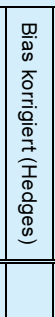 } & \multirow{2}{*}{ 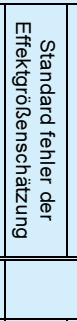 } & \multicolumn{2}{|c|}{$\begin{array}{l}\text { Konfidenzintervall für } \\
\text { die Effektgröße }\end{array}$} & \multirow{2}{*}{ 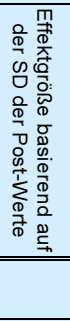 } & \\
\hline & Mittelwert & $n$ & SD & Mittelwert & $\mathrm{n}$ & SD & & & & $\begin{array}{l}\text { untere } \\
\text { Grenze }\end{array}$ & \begin{tabular}{|c|} 
obere \\
Grenze
\end{tabular} & & & & $\begin{array}{l}\text { untere } \\
\text { Grenze }\end{array}$ & $\begin{array}{l}\text { obere } \\
\text { Grenze }\end{array}$ & & \\
\hline \multicolumn{19}{|l|}{ Einzelkräuter } \\
\hline Chandola 1980 /C. tamala & 144.9 & 25 & 39.8 & 103.5 & 25 & 30.8 & 35.59 & 41.4 & 0.0002 & 21.2 & 61.6 & 1.2 & 1.1 & 0.3 & 0.5 & 1.7 & 1.3 & 1.00 \\
\hline $\begin{array}{l}\text { Kamble } 1998 \text { / Coccinia } \\
\text { indica }\end{array}$ & 365 & 15 & 37.2 & 112 & 15 & 11.6 & 27.55 & 253 & 0.0000 & 232.4 & 273.6 & 9.2 & 8.9 & 1.2 & 6.6 & 11.3 & 21.8 & 1.00 \\
\hline $\begin{array}{l}\text { Kuppurajan, } 1986 \text { / Coccinia } \\
\text { indica }\end{array}$ & 126.1 & 16 & 44 & 117.3 & 16 & 25.4 & 35.92 & 8.8 & 0.4937 & -17.1 & 34.7 & 0.2 & 0.2 & 0.4 & -0.5 & 0.9 & 0.3 & 0.19 \\
\hline $\begin{array}{l}\text { Kuppu Rajan } 1998 \text { / } \\
\text { Bockshornklee }\end{array}$ & 148 & 15 & 64.6 & 128.3 & 15 & 12.7 & 46.55 & 19.7 & 0.2563 & -15.1 & 54.5 & 0.4 & 0.4 & 0.4 & -0.3 & 1.1 & 1.6 & 0.43 \\
\hline Kumar 1999 / Bockshornklee & 174.6 & 51 & 37.1 & 121.3 & 51 & 26.6 & 32.28 & 53.3 & 0.0000 & 40.6 & 66.0 & 1.7 & 1.6 & 0.2 & 1.2 & 2.1 & 2.0 & 1.00 \\
\hline $\begin{array}{l}\text { Sharma 1996a / } \\
\text { Bockshornklee }\end{array}$ & 151.3 & 10 & 17.1 & 111.7 & 10 & 17.1 & 17.10 & 39.6 & 0.0001 & 23.5 & 55.7 & 2.3 & 2.2 & 0.6 & 1.1 & 3.3 & 2.3 & 1.00 \\
\hline $\begin{array}{l}\text { ICMR } 1998 \text { / Pterocarpus } \\
\text { marsupium }\end{array}$ & 151 & 93 & 17.3 & 119 & 93 & 23 & 20.35 & 32 & 0.0000 & 26.1 & 37.9 & 1.6 & 1.6 & 0.2 & 1.2 & 1.9 & 1.4 & 1.00 \\
\hline $\begin{array}{l}\text { Goyal and Tiwari, } 1999 / \\
\text { Vinca rosea }\end{array}$ & 131.4 & 25 & 30.2 & 92 & 25 & 18.2 & 24.93 & 39.4 & 0.0000 & 25.2 & 53.6 & 1.6 & 1.6 & 0.3 & 0.9 & 2.2 & 2.2 & 1.00 \\
\hline \multicolumn{19}{|l|}{ Kräutermischungen } \\
\hline Chowdhary 1998 / Ayush-82 & 169.3 & 89 & 35 & 144.2 & 89 & 30 & 32.60 & 25.1 & 0.0000 & 15.5 & 34.7 & 0.8 & 0.8 & 0.2 & 0.5 & 1.1 & 0.8 & 1.00 \\
\hline Kumar 1999/ Ayush-82 & 169.2 & 30 & 32.8 & 120.9 & 30 & 24.5 & 28.95 & 48.3 & 0.0000 & 33.3 & 63.3 & 1.7 & 1.6 & 0.3 & 1.1 & 2.2 & 2.0 & 1.00 \\
\hline Pandey 1995 / Ayush-82 & 193.1 & 80 & 48.6 & 135.8 & 80 & 41.4 & 45.14 & 57.3 & 0.0000 & 43.2 & 71.4 & 1.3 & 1.3 & 0.2 & 0.9 & 1.6 & 1.4 & 1.00 \\
\hline $\begin{array}{l}\text { Kumar } 1999 / \text { Abraga } \\
\text { chandraprabhavati }\end{array}$ & 168.3 & 30 & 28.5 & 119.2 & 30 & 31.3 & 29.93 & 49.1 & 0.0000 & 33.6 & 64.6 & 1.6 & 1.6 & 0.3 & 1.0 & 2.2 & 1.6 & 1.00 \\
\hline Shankar 1995 / Chendooram & 172.3 & 130 & 55 & 110 & 130 & 43.4 & 49.54 & 62.3 & 0.0000 & 50.2 & 74.4 & 1.3 & 1.3 & 0.1 & 1.0 & 1.5 & 1.4 & 1.00 \\
\hline Maji 1995 / D-400 & 208.5 & 19 & 12.1 & 113.1 & 19 & 6.9 & 9.85 & 95.4 & 0.0000 & 88.9 & 101.9 & 9.7 & 9.5 & 1.1 & 7.3 & 11.7 & 13.8 & 1.00 \\
\hline Sircar 1996 / MA-471 & 167.2 & 15 & 22 & 131.1 & 15 & 20 & 21.02 & 36.1 & 0.0001 & 20.4 & 51.8 & 1.7 & 1.7 & 0.4 & 0.8 & 2.5 & 1.8 & 1.00 \\
\hline Sircar 1996 / MA-471 & 115.8 & 30 & 20.5 & 106.3 & 30 & 24.3 & 22.48 & 9.5 & 0.1071 & -2.1 & 21.1 & 0.4 & 0.4 & 0.3 & -0.1 & 0.9 & 0.4 & 0.43 \\
\hline Sircar 1996 / MA-471 & 150.2 & 15 & 21.8 & 110.6 & 15 & 19.3 & 20.59 & 39.6 & 0.0000 & 24.2 & 55.0 & 1.9 & 1.9 & 0.4 & 1.0 & 2.7 & 2.1 & 1.00 \\
\hline Shankar 1994 / Sandana podia & 164.5 & 20 & 60.2 & 114.7 & 20 & 40.2 & 51.19 & 49.8 & 0.0039 & 17.0 & 82.6 & 1.0 & 1.0 & 0.3 & 0.3 & 1.6 & 1.2 & 0,92 \\
\hline
\end{tabular}

- Beide zum Zielparameter HbA1c untersuchte CCTs haben eine grosse ES (Bias korrigiert: 2,8 bzw. 1,0). Von den weiteren Studien hat nur eine Studie eine grosse ES $(1,0)$, die weiteren 4 Studien dieser Gruppe haben keine ES $(0,05)$, eine geringe ES $(0,4)$, und eine mittlere ES $(0,7$ und 0,8). Die Power ist bei 2 der 5 Studien nicht ausreichend (Tab. 5 und 6).

\section{Diskussion}

In vorherigen Veröffentlichungen wurde ayurvedischen Diabetesstudien eine allgemeine Underpowerung unterstellt (d.h. für einen Signifikanznachweis, z.B. aufgrund zu kleiner Patientenkollektive, nicht geeignet) [18]. Darüber hinaus besteht in der wissenschaftlichen Community vielfach die landläu- fige Meinung, Studien über die Wirksamkeit von CAM-Interventionen seien generell in den meisten Fällen unwissenschaftlich und „underpowered“ [4244]. Dies gipfelte in einem Artikel aus dem New England Journal of Medicine von 1998, in dem die Forderung laut wurde, CAM-Verfahren auf der Basis des vermeintlichen Unwissenschaftlichkeitspostulates weitestgehend aus der medizinischen Versorgung zu verbannen: „It is time for the scientific community to stop giving alternative medicine a free ride" [45].

Diese Behauptungen treffen zumindest für die hier betrachteten ayurvedischen Diabetes-Studien nicht oder nur zu einem kleinen Teil zu. Design, Effektstärke und Power der untersuchten Studien wurde im Rahmen dieser Arbeit eingehend untersucht. Mehr als $75 \%$ der randomisierten, kontrollierten bzw. kontrollierten Studien hatten eine grosse Effektstärke (ES>0,8) bzgl. Nüchternblutzucker, postprandialem Blutzucker und HbA1c. Das Gleiche gilt für die nicht-kontrollierten Studien mit Einzelkräutern oder Kräutermischungen. Mehr als 70\% der untersuchten Studien weisen eine ausreichende Power auf. Aussagen zur Wirksamkeit ayurvedischer Diabetes-Intervention sind auf der Basis der vorhandenen Evidenzen also durchaus möglich. Dennoch sind weitere randomisierte, kontrollierte Studien zu fordern: Studien mit kleinen Studiengruppen sind zwangsläufig anfälliger für methodische und statistische Fehler verschiedener Art (Kleine Patientenkollektive können bei Ausreissern oder falschen Messungen schon eines einzigen Patienten zu erheblichen Ergebnisverzerrungen führen). Zudem erschwert eine mitunter lückenhafte Auswertung bzw. Dokumentation eine umfassende Metaanalyse. 
Tab. 3. (Randomisierte) kontrollierte klinische Studien: Studiengrösse, Mittelwerte vor und nach der Behandlung, Standardabweichungen, Vergleich Phytoarm mit Kontrollarm(unverbundener T-Test), Effektgrösse und Power: Postprandialer Blutzucker (mg/dl)

\begin{tabular}{|c|c|c|c|c|c|c|c|c|c|c|c|c|c|c|c|c|c|c|c|}
\hline \multirow{3}{*}{ Studie } & \multicolumn{7}{|c|}{ BASISDATEN } & \multicolumn{5}{|c|}{$\begin{array}{l}\text { DIFFERENZ DER } \\
\text { DIFFERENZEN }\end{array}$} & \multicolumn{6}{|c|}{$\begin{array}{c}\text { STANDARDISIERTE } \\
\text { EFFEKTGRÖSSE }\end{array}$} & \multirow{2}{*}{ 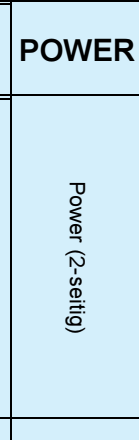 } \\
\hline & \multicolumn{4}{|c|}{ Phytoarm } & \multicolumn{3}{|c|}{ Kontrollarm } & 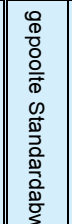 & 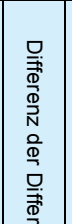 & 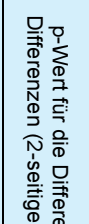 & \multicolumn{2}{|c|}{$\begin{array}{c}95 \% \\
\text { Konfidenzinter- } \\
\text { vall fur die } \\
\text { Differenz der } \\
\text { Differenzen }\end{array}$} & \multirow[t]{2}{*}{ 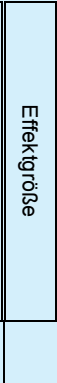 } & \multirow{2}{*}{ 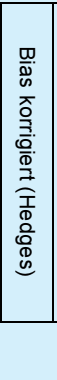 } & \multirow{2}{*}{ 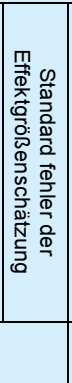 } & \multicolumn{2}{|c|}{$\begin{array}{l}\text { Konfidenzinter- } \\
\text { vall für die } \\
\text { Effektgröße }\end{array}$} & \multirow{2}{*}{ 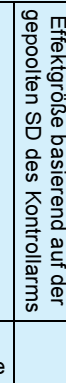 } & \\
\hline & & Mittelwert & $n$ & SD & Mittelwert & $n$ & SD & & & & $\begin{array}{l}\text { untere } \\
\text { Grenze }\end{array}$ & $\begin{array}{c}\text { obere } \\
\text { Grenze }\end{array}$ & & & & \begin{tabular}{l|} 
untere \\
Grenze
\end{tabular} & $\begin{array}{l}\text { obere } \\
\text { Grenze }\end{array}$ & & \\
\hline \multicolumn{20}{|l|}{$\begin{array}{l}\text { Randomisierte Kontrollierte } \\
\text { Studien }\end{array}$} \\
\hline \multirow{2}{*}{ Azad Khan 1979 / Coccinia indica } & Prä & 245.4 & 16 & 41.4 & 255.1 & 16 & 58.6 & \multirow{2}{*}{55.7} & \multirow{2}{*}{55.4} & \multirow{2}{*}{0.0086} & \multirow{2}{*}{15.2} & \multirow{2}{*}{95.6} & \multirow{2}{*}{1.0} & \multirow{2}{*}{1.0} & \multirow{2}{*}{0.4} & \multirow{2}{*}{0.2} & \multirow{2}{*}{1.7} & \multirow{2}{*}{0.9} & \multirow[t]{2}{*}{0.86} \\
\hline & Post & 186.9 & 16 & 54.9 & 252 & 16 & 63.4 & & & & & & & & & & & & \\
\hline Agrawal 1996 / Ocimum sanctum & \begin{tabular}{|c|} 
Prä \\
Post
\end{tabular} & $\frac{223.9}{204}$ & 20 & $\begin{array}{c}19.9 \\
25\end{array}$ & $\begin{array}{l}221.6 \\
215.1\end{array}$ & \begin{tabular}{|l|}
20 \\
20
\end{tabular} & \begin{tabular}{|l|}
20.1 \\
27.1
\end{tabular} & 23.5 & 13.4 & 0.0792 & -1.6 & 28.4 & 0.6 & 0.6 & 0.3 & -0.1 & 1.2 & 0.6 & 0.55 \\
\hline \begin{tabular}{|l|}
$\begin{array}{l}\text { Kontrollierte Klinische } \\
\text { Studien }\end{array}$ \\
\end{tabular} & & & & & & & & & & & & & & & & & & & \\
\hline Kamble 1996 / Coccinia indica & Prä & 308 & 25 & 24.5 & 292 & 15 & 28.1 & 22.9 & 23.0 & 0.0039 & 7.8 & 38.2 & 1.0 & 1.0 & 0.3 & 0.3 & 1.7 & 0.9 & 0.91 \\
\hline 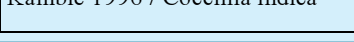 & Post & 145 & 25 & 14.1 & 152 & 15 & 20.2 & ]$^{22.3}$ & 25.0 & 0.0053 & 1.0 & 5.2 & 100 & 1.0 & 0.0 & 0.0 & 1., & & 0.31 \\
\hline
\end{tabular}

Tab. 4. Einzelkräuter und Kräutermischungen: Studiengrösse, Mittelwerte vor und nach der Behandlung, Standardabweichungen, verbundener T-Test, Effektgrösse und Power: Postprandialer Blutzucker (mg/dl)

\begin{tabular}{|c|c|c|c|c|c|c|c|c|c|c|c|c|c|c|c|c|c|c|}
\hline \multirow{3}{*}{ Studie } & \multicolumn{6}{|c|}{ BASISDATEN } & \multicolumn{5}{|c|}{ DIFFERENZ } & \multicolumn{6}{|c|}{$\begin{array}{c}\text { STANDARDISIERTE } \\
\text { EFFEKTGRÖSSE }\end{array}$} & \multirow{3}{*}{ 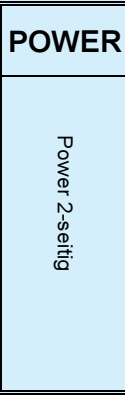 } \\
\hline & \multicolumn{3}{|c|}{ Prä } & \multicolumn{3}{|c|}{ Post } & \multirow{2}{*}{ 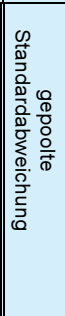 } & \multirow{2}{*}{ 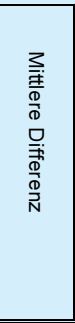 } & \multirow{2}{*}{ 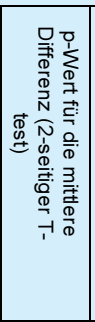 } & \multicolumn{2}{|c|}{$\begin{array}{l}95 \% \\
\text { Konfidenzintervall } \\
\text { für die Differenz }\end{array}$} & \multirow[t]{2}{*}{ 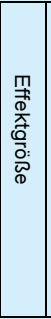 } & \multirow{2}{*}{ 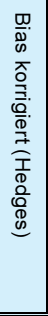 } & \multirow{2}{*}{ 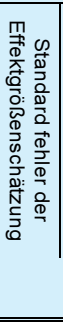 } & \multicolumn{2}{|c|}{$\begin{array}{l}\text { Konfidenzintervall } \\
\text { für die Effektgröße }\end{array}$} & \multirow{2}{*}{ 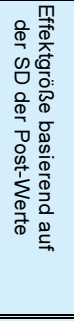 } & \\
\hline & Mittelwert & $\mathrm{n}$ & SD & Mittelwert & $\mathrm{n}$ & SD & & & & $\begin{array}{l}\text { untere } \\
\text { Grenze }\end{array}$ & $\begin{array}{c}\text { obere } \\
\text { Grenze } \\
\end{array}$ & & & & $\begin{array}{l}\text { untere } \\
\text { Grenze } \\
\end{array}$ & \begin{tabular}{|c|} 
obere \\
Grenze \\
\end{tabular} & & \\
\hline \multicolumn{19}{|l|}{ Einzelkräuter } \\
\hline Chandola 1980 /C. tamala & 236.2 & 25 & 72.1 & 170.6 & 25 & 54.7 & 64.0 & 65.6 & 0.001 & 29.2 & 102.0 & 1.0 & 1.0 & 0.3 & 0.4 & 1.6 & 1.2 & 0,92 \\
\hline $\begin{array}{l}\text { Kuppurajan, } 1986 \text { / Coccinia } \\
\text { indica }\end{array}$ & 233.1 & 16 & 107.2 & 197.7 & 16 & 23.3 & 77.6 & 35.4 & 0.207 & -20.6 & 91.4 & 0.5 & 0.4 & 0.4 & -0.3 & 1.1 & 1.5 & 0.46 \\
\hline $\begin{array}{l}\text { Kuppu Rajan } 1998 \text { / } \\
\text { Bockshornklee }\end{array}$ & 314.3 & 15 & 25.2 & 264.8 & 15 & 21.3 & 23.3 & 49.5 & 0.000 & 32.0 & 67.0 & 2.1 & 2.1 & 0.5 & 1.2 & 3.0 & 2.3 & 1.00 \\
\hline Kumar 1999 / Bockshornklee & 236.5 & 51 & 39.2 & 183.8 & 51 & 16.9 & 30.2 & 52.7 & 0.000 & 40.8 & 64.6 & 1.7 & 1.7 & 0.2 & 1.3 & 2.2 & 3.1 & 1.00 \\
\hline $\begin{array}{l}\text { Sharma 1996a / } \\
\text { Bockshornklee }\end{array}$ & 257.6 & 10 & 34.2 & 171.1 & 10 & 34.2 & 34.2 & 86.5 & 0.000 & 54.4 & 118.6 & 2.5 & 2.4 & 0.6 & 1.3 & 3.6 & 2.5 & 1.00 \\
\hline $\begin{array}{l}\text { ICMR } 1998 \text { / Pterocarpus } \\
\text { marsupium }\end{array}$ & 216 & 93 & 21.5 & 171 & 93 & 29.1 & 25.6 & 45.0 & 0.000 & 37.6 & 52.4 & 1.8 & 1.8 & 0.2 & 1.4 & 2.1 & 1.5 & 1.00 \\
\hline $\begin{array}{l}\text { Goyal and Tiwari, } 1999 \text { / } \\
\text { Vinca rosea }\end{array}$ & 224.9 & 25 & 22.5 & 144.2 & 25 & 27.3 & 25.0 & 80.7 & 0.000 & 66.5 & 94.9 & 3.2 & 3.2 & 0.4 & 2.3 & 4.0 & 3.0 & 1.00 \\
\hline \multicolumn{19}{|l|}{ Kräutermischungen } \\
\hline Chowdhary 1998 / Ayush-82 & 249.6 & 89 & fehlt & 219.2 & 89 & fehlt & & & & & & & & & & & & \\
\hline Kumar 1999 / Ayush-82 & 218.4 & 30 & 38.6 & 172.9 & 30 & 34.2 & 36.5 & 45.5 & 0.000 & 26.7 & 64.3 & 1.2 & 1.2 & 0.3 & 0.7 & 1.8 & 1.3 & 1.00 \\
\hline Pandey 1995 / Ayush-82 & 283.7 & 80 & 91.2 & 202.6 & 80 & 45.9 & 72.2 & 81.1 & 0.000 & 58.6 & 103.6 & 1.1 & 1.1 & 0.2 & 0.8 & 1.5 & 1.8 & 1.00 \\
\hline $\begin{array}{l}\text { Kumar } 1999 \text { / Abraga } \\
\text { chandraprabhavati }\end{array}$ & 234.1 & 30 & 43.3 & 181.8 & 30 & 14 & 32.2 & 52.3 & 0.000 & 35.7 & 68.9 & 1.6 & 1.6 & 0.3 & 1.0 & 2.2 & 3.7 & 1.00 \\
\hline Shankar 1995 / Chendooram & 267.7 & 140 & 73.5 & 183 & 140 & 63.1 & 68.5 & 84.7 & 0.000 & 68.6 & 100.8 & 1.2 & 1.2 & 0.1 & 1.0 & 1.5 & 1.3 & 1.00 \\
\hline Maji 1995 / D-400 & 262 & 19 & 20.1 & 141.2 & 19 & 8.3 & 15.4 & 120.8 & 0.000 & 110.7 & 130.9 & 7.9 & 7.7 & 0.9 & 5.8 & 9.5 & 14.6 & 1.00 \\
\hline Sircar 1996 / MA-471 & 290 & 15 & 28.3 & 230.9 & 15 & 23.1 & 25.8 & 59.1 & 0.000 & 39.8 & 78.4 & 2.3 & 2.2 & 0.5 & 1.3 & 3.1 & 2.6 & 1.00 \\
\hline Sircar 1996 / MA-471 & 172 & 30 & 19.3 & 163.9 & 30 & 18.4 & 18.9 & 8.1 & 0.102 & -1.6 & 17.8 & 0.4 & 0.4 & 0.3 & -0.1 & 0.9 & 0.4 & 0.56 \\
\hline Sircar 1996 / MA-471 & 247.3 & 15 & 22.3 & 186.1 & 15 & 18 & 20.3 & 61.2 & 0.000 & 46.0 & 76.4 & 3.0 & 2.9 & 0.5 & 1.9 & 4.0 & 3.4 & 1.00 \\
\hline $\begin{array}{l}\text { Shankar } 1994 \text { / Sandana } \\
\text { podia }\end{array}$ & 281.7 & 20 & 95.7 & 171.3 & 20 & 72.2 & 84.8 & 110.4 & 0.000 & 56.1 & 164.7 & 1.3 & 1.3 & 0.3 & 0.6 & 2.0 & 1.5 & 0.99 \\
\hline $\begin{array}{l}\text { Sivaprakasam et al. 1994, } \\
\text { Shudh Shilajitu }\end{array}$ & 303.9 & 25 & 88.4 & 208.1 & 25 & 77.8 & 83.3 & 95.8 & 0.000 & 48.4 & 143.2 & 1.2 & 1.1 & 0.3 & 0.5 & 1.7 & 1.2 & 0.99 \\
\hline
\end{tabular}


Tab. 5. Kontrollierte klinische Studien: Studiengrösse, Mittelwerte vor und nach der Behandlung, Standardabweichungen, Vergleich Phytoarm mit Kontrollarm, (unverbundener T-Test), Effektgrösse und Power: HbA1c (\%)

\begin{tabular}{|c|c|c|c|c|c|c|c|c|c|c|c|c|c|c|c|c|c|c|c|}
\hline \multirow{3}{*}{ Studie } & \multicolumn{7}{|c|}{ BASISDATEN } & \multicolumn{5}{|c|}{$\begin{array}{c}\text { DIFFERENZ DER } \\
\text { DIFFERENZEN }\end{array}$} & \multicolumn{6}{|c|}{$\begin{array}{c}\text { STANDARDISIERTE } \\
\text { EFFEKTGRÖSSE }\end{array}$} & \multirow{3}{*}{ 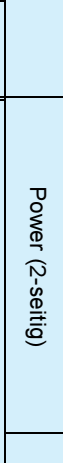 } \\
\hline & \multicolumn{4}{|c|}{ Phytoarm } & \multicolumn{3}{|c|}{ Kontrollarm } & \multirow{2}{*}{ 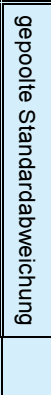 } & \multirow{2}{*}{ 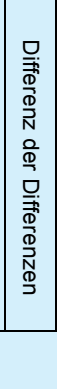 } & \multirow{2}{*}{ 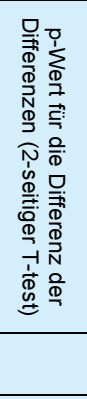 } & \multicolumn{2}{|c|}{$\begin{array}{c}95 \% \\
\text { Konfidenzinter- } \\
\text { vall für die } \\
\text { Differenz der } \\
\text { Differenzen }\end{array}$} & \multirow[t]{2}{*}{ 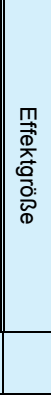 } & \multirow{2}{*}{ 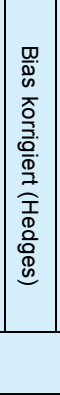 } & \multirow{2}{*}{ 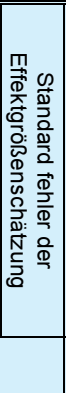 } & \multirow{2}{*}{\multicolumn{3}{|c|}{ 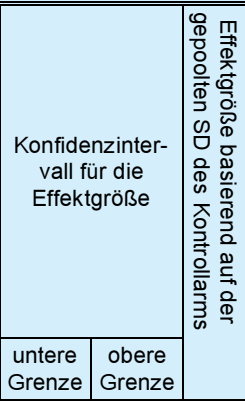 }} & \\
\hline & & Mittelwert & $\mathrm{n}$ & SD & Mittelwert & $n$ & SD & & & & \begin{tabular}{l|} 
untere \\
Grenze
\end{tabular} & $\begin{array}{c}\text { obere } \\
\text { Grenze }\end{array}$ & & & & & & & \\
\hline \multicolumn{20}{|l|}{$\begin{array}{l}\text { KONTROLLIERTE KLINISCHE } \\
\text { STUDIEN }\end{array}$} \\
\hline \multirow{2}{*}{ Baskaran 1990 / Gymnema sylvestre } & Prä & 11.9 & 22 & 1.4 & 10.2 & 25 & 0.8 & \multirow[t]{2}{*}{0.9} & \multirow[t]{2}{*}{2.5} & \multirow{2}{*}{0.0000} & \multirow{2}{*}{2.0} & \multirow{2}{*}{3.1} & \multirow{2}{*}{2.8} & \multirow{2}{*}{2.7} & \multirow{2}{*}{0.4} & \multirow{2}{*}{1.9} & \multirow{2}{*}{3.5} & \multirow[t]{2}{*}{3.4} & \multirow{2}{*}{1} \\
\hline & Post & 9.6 & 22 & 1.0 & 10.5 & 25 & 0.7 & & & & & & & & & & & & \\
\hline \multirow{2}{*}{$\begin{array}{l}\text { Shanmugasundaram } 1990 \text { / Gymnema } \\
\text { sylvestre }\end{array}$} & Prä & 12.8 & 27 & 2.0 & 12.7 & 37 & 2.1 & \multirow{2}{*}{2.4} & \multirow{2}{*}{2.4} & \multirow{2}{*}{0.0002} & \multirow{2}{*}{1.2} & \multirow{2}{*}{3.6} & 1.0 & 1.0 & 0.3 & 0.5 & 1.5 & 1.0 & 0.97 \\
\hline & Post & 9.5 & 27 & 1.7 & 11.8 & 37 & 2.4 & & & & & & 100 & 1.0 & 0.3 & 0.5 & 1.0 & 1.0 & 10. \\
\hline
\end{tabular}

Tab. 6. Einzelkräuter und Kräutermischungen: Studiengrösse, Mittelwerte vor und nach der Behandlung, Standardabweichungen, verbundener T-Test, Effektgrösse und Power: HbA1c (\%)

\begin{tabular}{|c|c|c|c|c|c|c|c|c|c|c|c|c|c|c|c|c|c|c|}
\hline \multirow{3}{*}{ Studie } & \multicolumn{6}{|c|}{ BASISDATEN } & \multicolumn{5}{|c|}{ DIFFERENZ } & \multicolumn{6}{|c|}{$\begin{array}{l}\text { STANDARDISIERTE } \\
\text { EFFEKTGRÖSSE }\end{array}$} & \multirow{3}{*}{ 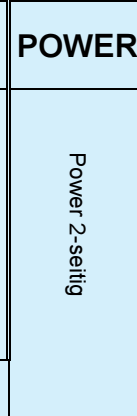 } \\
\hline & \multicolumn{3}{|c|}{ Prä } & \multicolumn{3}{|c|}{ Post } & \multirow{2}{*}{ 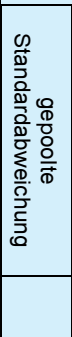 } & \multirow{2}{*}{ 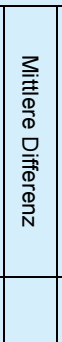 } & \multirow{2}{*}{ 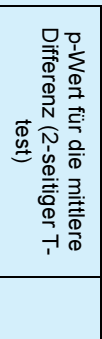 } & \multicolumn{2}{|c|}{$\begin{array}{c}95 \% \\
\text { Konfidenzintervall } \\
\text { für die Differenz }\end{array}$} & \multirow{2}{*}{ 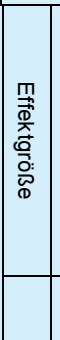 } & \multirow{2}{*}{ 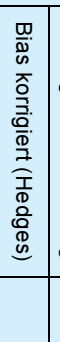 } & \multirow{2}{*}{ 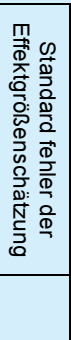 } & \multicolumn{2}{|c|}{$\begin{array}{l}\text { Konfidenzintervall } \\
\text { für die } \\
\text { Effektgröße }\end{array}$} & \multirow{2}{*}{ 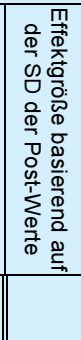 } & \\
\hline & Mittelwert & $n$ & SD & Mittelwert & $n$ & SD & & & & \begin{tabular}{|c|} 
untere \\
Grenze
\end{tabular} & \begin{tabular}{|c|} 
obere \\
Grenze
\end{tabular} & & & & \begin{tabular}{|c|} 
untere \\
Grenze
\end{tabular} & \begin{tabular}{|c||} 
obere \\
Grenze
\end{tabular} & & \\
\hline \multicolumn{19}{|l|}{ Einzelkräuter } \\
\hline $\begin{array}{l}\text { Sharma 1996/ } \\
\text { Bockshornklee }\end{array}$ & 9.6 & 10 & 1.9 & 8.4 & 10 & 1.4 & 1.7 & 1.2 & 0.1253 & -0.4 & 2.8 & 0.7 & 0.7 & 0.5 & -0.2 & 1.6 & 0.9 & 0.51 \\
\hline $\begin{array}{l}\text { ICMR } 1998 \text { / Pterocarpus } \\
\text { marsupium }\end{array}$ & 9.8 & 67 & 1 & 9.4 & 67 & 0.9 & 1.0 & 0.4 & 0.0163 & 0.1 & 0.7 & 0.4 & 0.4 & 0.2 & 0.1 & 0.8 & 0.4 & 0.90 \\
\hline \multicolumn{19}{|l|}{ Kräutermischungen } \\
\hline Sircar $1996 /$ MA-471 & 10.3 & 15 & 2.8 & 8.5 & 15 & 1.5 & 2.2 & 1.8 & 0.0364 & 0.1 & 3.5 & 0.8 & 0.8 & \begin{tabular}{|l|}
0.4 \\
\end{tabular} & 0.0 & 1.5 & 1.2 & 0.82 \\
\hline Sircar 1996 / MA-471 & 7 & 30 & 2.0 & 7.1 & 30 & 2.1 & 2.0 & 0.1 & 0.8498 & -1.0 & 1.2 & 0.0 & 0.0 & 0.3 & -0.5 & 0.6 & 0.0 & 0.05 \\
\hline Sircar 1996 / MA-471 & 10.1 & 15 & 2.5 & 8 & 15 & 1.7 & 2.1 & 2.1 & 0.0119 & 0.5 & 3.7 & 1.0 & 1.0 & 0.4 & 0.2 & 1.7 & 1.2 & 0.95 \\
\hline
\end{tabular}

Für zukünftige Studien sind, basierend auf beobachteten methodischen Mängeln der vorhandenen Evidenz deshalb in erster Linie folgende Anstrengungen $\mathrm{zu}$ fordern:

- Insgesamt sollten Studiendesign, Rekrutierungsverfahren und Auswertungsmethoden transparenter, vollständiger und entsprechend den Grundsätzen der „guten klinischen und statistischen Praxis“ dokumentiert werden. Eine Studie sollte neben Vollständigkeit vor allem auf Verständlichkeit, Konsistenz und Plau- sibilität optimiert sein. Besonders wichtig sind dabei:

- eine präzise Beschreibung der Fragestellung und Klarheit der Studienziele.

- eine präzise operationalisierte Definition der Haupt- und Nebenzielkriterien mit Angabe zur wissenschaftlichen Aktualität und Akzeptanz der verwendeten Messverfahren.

- eine der Fragestellung entsprechende Wahl des Studiendesigns.

- eine der Fragestellung entspre- chende Wahl der Studienpopulation (Ein- und Ausschlusskriterien).

- eine nachvollziehbare der Fragestellung und der geplanten Auswertung entsprechende Fallzahlplanung unter Angabe des $\alpha$ - und $\beta$-Niveaus und der Grösse der medizinischen Relevanz.

- Die Drop-out-Rate ist anzugeben.

- Angabe einer zumindest in den Grundzügen formulierten Auswertungsstrategie für die Haupt und Nebenzielkriterien unter Angabe der statistischen Testmethoden und 
der Verfahren zum Umgang mit fehlenden Daten und Drop-outs [46-48].

- Traditionelle ayurvedische Therapiestrategien bei Diabetes mellitus sind fast immer multimodal/multidisziplinär. So beinhalten sie neben phytotherapeutischen beispielsweise ausleitende Verfahren und manuelle Therapiemethoden. Die Ergebnisse der klinischen Forschung bisher fast ausschliesslich auf phytotherapeutische Interventionen beschränkt - bilden das therapeutische Spektrum des Ayurveda bei Diabetes mellitus deswegen nur teilweise ab. Für eine realistischere Darstellung müssen zukünftig auch andere Therapiesäulen systematisch untersucht werden. Nur so ist es möglich, den von Seiten des Ayurveda postulierten Synergieeffekt der neben- und miteinander eingesetzten Therapieelemente $\mathrm{zu}$ untersuchen.

- Es fällt auf, dass im Bereich der Phytotherapie die Gewichtung der einzelnen Heilpflanzen in der klinischen Forschung nicht immer mit der Bedeutung der Heilpflanzen in der modernen ayurvedischen Praxis/den klassischen Empfehlungen des Ayurveda korreliert. Eine nicht geringe Zahl untersuchter Pflanzen spielt in der Praxis eine eher untergeordnete Rolle (z.B. Gymnema sylvestre und Coccinia indica). Andere häufig verwendete Heilpflanzen sind in der klinischen Forschung hingegen unterrepräsentiert (z.B. Curcuma longa, Tinospora cordifolia oder Azadirachta indica). Im Sinne einer orientierenden Bewertung ayurvedischer Interventionen wäre es deshalb sinnvoll, den Forschungsschwerpunkt zunächst auf die therapeutischen Kernbereiche des Ayurveda zu richten.

- Die Zielparameter wurden in der Regel nur einmal bestimmt. Zur Fehlerminimierung sollten gerade in diesen Bereichen mehrere Messungen stattfinden. Hier handelt es sich um einen Bias: die Vermeidung (oder zumindest Reduktion) einer Verfälschung der Aussage (Verzerrung) durch Störfaktoren (confounding) und betrifft alle Faktoren mit Einfluss auf die Zielgrösse, die mit

\section{Stratifizierte Randomisierte Studien Ansatz für die Konstitutionsmedizin Ayurveda}

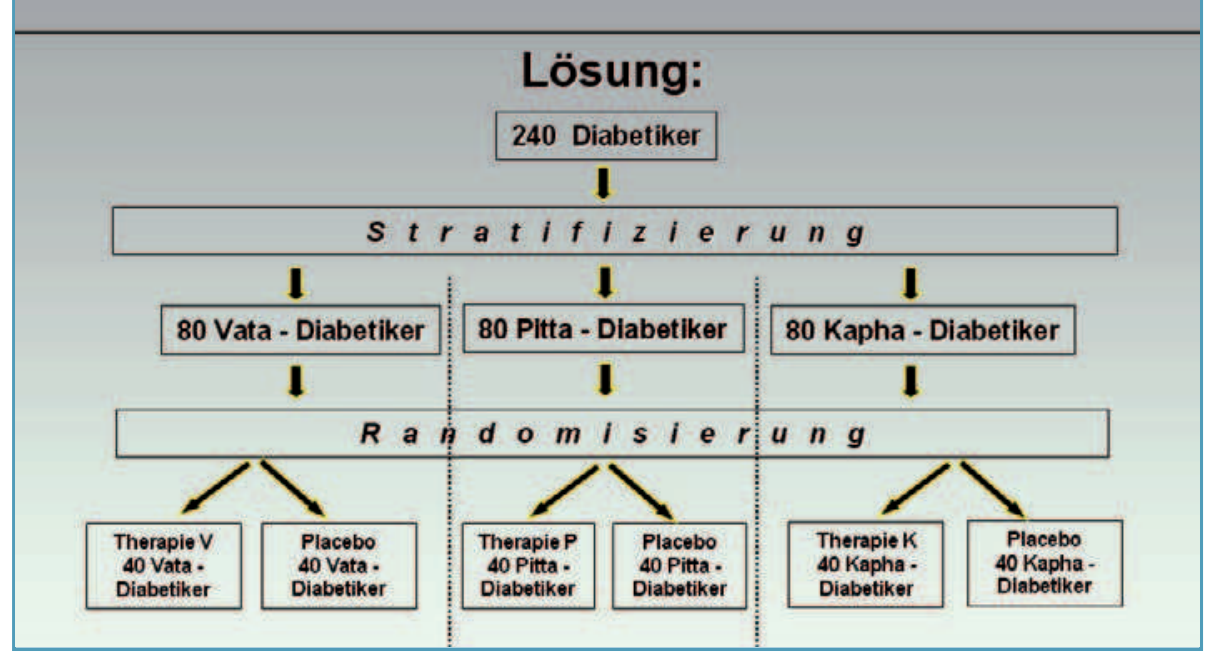

Abb. 3. Stratifizierte Randomisierung: Beispiel-Design.

der Behandlung wechselwirken und so Einfluss nehmen können (z.B. falsche Eichung, falsche/ungenaue Messverfahren, Zeitdifferenz, heterogene Gruppen, falsche Behandlung, Arzt-Einfluss). Dies erfordert Massnahmen:

- bei der Planung: Randomisation, Stratifikation

- bei der Auswertung: Post-Stratifikation

- Regressionsmodelle

- Varianzanalysen mit Interaktionen

- Methoden (Messungen) normieren

- Ayurveda ist eine Konstitutionsmedizin. Individuellen Diagnose- und Therapiekriterien wird ein sehr hoher Stellenwert beigemessen. Eine Integration ayurvedischer Diagnoseund Bewertungsverfahren in die Studiendesigns, beispielsweise die Einteilung von Patienten verschiedener Konstitutionstypen in entsprechende Studienarme, könnte den besonderen Eigenschaften der ayurvedischen Medizin in der klinischen Forschung besser Rechnung tragen. Lösungsansätze bieten methodische Ansätze, die zu einer verbesserten Anpassung an ayurvedische Intervention führen:

\section{- Stratifizierte Randomisierung}

Die klassische randomisierte Studie ist im Bereich Ayurveda oft problematisch: Bei einem identischen Krankheitsbild werden in

\begin{abstract}
Abhängigkeit von der Konstitution des Patienten individuelle, z.T. sehr unterschiedliche Therapien eingesetzt. Diese Tatsache findet im Rahmen eines klassischen RCTs jedoch keine Berücksichtigung. Im Gegenteil: Durch das Poolen der Studienteilnehmer erhalten alle Teilnehmer in der Studiengruppe nicht nur die gleiche Therapie, sondern - aus ayurvedischer Perspektive - möglicherweise sogar eine für sie kontraindizierte Therapie. Dies kann z.B. zu Fehlern der 2. Art ( $\beta$ ) führen, möglicherweise sogar zu einer Ablehnung der untersuchten Intervention. Subgruppenanalysen bzw. Stratifizierungen der Studienteilnehmer entsprechend ihres ayurvedischen Konstitutionstyps könnten mit grosser Wahrscheinlichkeit $\mathrm{zu}$ einem anderen Ergebnis führen (Abb. 3).
\end{abstract}

Zusammenfassend kann gesagt werden, dass bei mehr als 45 verschiedenen Behandlungsformen des Typ-2Diabetes mellitus die Anzahl der randomisierten, klinischen Studien pro Verfahren für eine abschliessende Bewertung nach den Prinzipien der Evidenz-basierten Medizin - trotz einer Anzahl viel versprechender Einzelund Kombinationspräparate - derzeit noch zu gering ist. 
Durch insgesamt höherwertigere Wirksamkeitsstudien mit mulitzentrischen und multinationalen Studiendesigns auf der Basis externer Bewertung (peer-reviewed) könnte das Evidenzniveau schnell erhöht werden. Grundsätzlich gilt, dass für eine Übernahme ausgewählter ayurvedischer Therapien in westliche Gesundheitssysteme noch wesentliche Voraussetzung geschaffen werden müssen. Gerade wegen seit Jahren ständig steigender Nachfrage nach Therapien des Ayurveda sollten besonders erfolgversprechende Therapiemethoden nach dem Motto „die beste Therapie gewinnt“ in einem zweistufigen Prozess nicht nur innerhalb des ayurvedischen Medizinsystems miteinander verglichen oder gegen Placebo getestet werden, sondern im nächsten Schritt auch konsequent mit vergleichbaren Therapien aus der etablierten Medizin verglichen werden. So könnte es mittel- und langfristig gelingen, die konventionellen Behandlungsstrategien, insbesondere im Problembereich chronischer Erkrankungen, um evidenzbasierte ayurvedische Therapien zu ergänzen.

\section{Literatur}

1. World Health Organization: Traditional Medicine in Asia. WHO Regional Publications, South-EastAsia Series, No. 39, WHO-Verlag, 2002a.

2. Indian Systems of Medicine \& Homoeopathy (2004). http://www.indianmedicine.nic.in.

3. Ranade SB: Ayurveda - Wesen und Methodik. Haug Verlag, Heidelberg 2004.

4. Caraka Samhita, Vimana Sthana IV.12: Text with English Translation and Critical Exposition based on Cakrapani Datta's Ayurveda Dipika by Sharma RK, Dash B. 7 Bände. Chaukhamba, Neu Delhi 2001.

5. Sushruta Samhita: With English Translation of Text and Dalhana's Commentary along with Critical Notes/edited and translated by Priya Vrat Sharma: Chaukhamba Visvabharati, Varanasi 2001.

6. Astangahrdaya Samhita of Vagbhata: The Book of Eight Branches of Ayurveda/translated by a Board of Scholars. 3 Bd. Vedams, Delhi 1999

7. Singh $\mathrm{RH}$ : The holistic principles of ayurvedic medicine. Chaukhamba, Neu Delhi 2003.

8. Zimmermann F: Gentle Purge. The Flower Power of Ayurveda. Charles Leslie and Allan Young (Hrsg.), Path to Asian Medical Knowledge; University Press of California, Berkeley etc. 1992: 209-223.

9. Pubmed-Datenbank (2005). http://www.ncbi.nlm.nih.gov.

10. Apitz R, Winter S: Potenziale und Ansätze der Prävention - aktuelle Entwicklungen in Deutschland. Der Internist 2004;45(2):139-47.

11. Brenner G, Altenhofen L, Knoepnadel J, Weber I: Nationale Gesundheitsziele: Diabetes mellitus Typ 2 als Zielbereich, Bundesgesundheitsblatt 2, 2003.

12. Khajuria S, Thomas J: Traditional Indian beliefs about the dietary management of diabetes B. An exploratory study of the implications for the management of Gujarati diabetics in Britain. Hum Nutr Diet 1992;5(5):311-321.

13. Shah V: Diabetes mellitus in Indian medicine Chaukhambha Orientalia, Varansi (India) 1995

14. Mishra L, Singh B, Dagenais S: Healthcare and disease management in Ayurveda. Altern The Health Med 2001:7:44-50

15. Atharvale VB: Pathogenesis in Ayurveda. Motilal Banarsidass, Neu Delhi 2001.

16. Madhava Nidanam (Roga Viniscaya) of Mahavakara (A Treatise On Ayurveda). Translated into English by Srikantha Murthy. Nachdruck. Chaukambha Orientalia, Varanasi 2001.

17. Murty S: Doctrines of Pathology in Ayurveda Vedams, Neu Delhi 1998.

18. Hardy et al.: Report/Technology Assessment Number 41: Ayurvedic Interventions for Diabetes mellitus: A Systematic Review. AHRQ Publication No. 01-E040, Rockville, USA, 2001

19. Elder C: Ayurveda for Diabetes mellitus: A Review of the biomedical literature. Alternative Therapies 2004;10(1):44-50.

20. Jadad AR, Moor AR, Carroll D et al.: Assessing the quality of reports of randomized clinical trials: Is blinding necessary? Control Clin Trials 1996: 17:1-12.

21. Azad Khan AK, Akhtar S, Mahtab H: Coccinia indica in the treatment of patients with diabetes mellitus. Bangladesh Med Res Counc Bul 1979;5(2):60-6.

22. Agrawal $P$, Rai $V$, Singh RB: Randomized placebo-controlled single-blind trial of holy basil leaves in patients with non-insulin-dependent diabetes mellitus. Int J Clin Pharmacol Ther 1996;34 406-409.

23. Chandola HM, Tripathi SN, Udupa KN: Variations in the progression of maturity onset diabetes according to body constitution. Ancient Sci Life 1994;13(3\&4):293-301.

24. Kamble SM, Jyotishi GS, Kamalakar PL et al. Efficacy of Coccinia indica W. \& A. in diabetes mellitus. J Res Ayurveda and Siddha 1996; XVII(1-2):77-84.

25. Kohli $K R$, Singh $\mathrm{RH}$. A clinical trial of jambu (Eugenia jambolana) in non-insulin dependant diabetes mellitus. J Res Ayurveda and Siddha 1993 Sept-Dec 31;XIV(304):89-97.

26. Baskaran K, Ahamath BK, Shanmugasundaram KR et al.: Antidiabetic effect of a leaf extract from Gymnema sylvestre in non-insulin-dependent diabetes mellitus patients. J Ethnopharmacol 1990;30(3):295-300.

27. Shanmugasundaram ER, Gopinath KL, Shanmugasundaram KR et al.: Possible regeneration of the islets of Langerhans in streptozotocin-diabetic rats given Gymnema sylvestre lea extracts. J Ethnopharmacol 1990a;30:265-279.

28. Shanmugasundaram ER, Rajeswari G, Bas-karan K et al.: Use of Gymnema sylvestre leaf extract in the control of blood glucose in insulin-dependent diabetes mellitus. J Ethnopharmaco 1990b;30(3):281-94

29. Chandola HM, Tripathi SN, Udupa KN: Hypo-glycaemic response of $\mathrm{C}$. tamala in patients of maturity onset (insulin independent) diabetes. Res Ayurveda and Siddha 1980a;1(2):275-290.

30. Kamble SM, Kamlakar $\mathrm{PL}$, Vaidya $\mathrm{S}$ et al. Influence of Coccinia indica on certain enzymes in glycolytic and lipolytic pathway in human diabetes. Indian J Med Sci 1998;52(4):143-146.

31. Kuppurajan K, Seshadri C, Revathi R et al.: Hypoglycaemic effect of Coccinia indica in diabetes mellitus. Nagarjun 1986;29(5):1-4.

32. Kuppu Rajan K, Srivatsa A, Krishnaswami CV et al.: Hypoglycemic and hypotriglyceridemic effects of methika churna (fenugreek). Antiseptic 1998;95(3):78-79.

33. Kumar N, Kumar A, Sharma ML: Clinical evaluation of single and herbo-mineral compound drugs in the management of madhumeha. J Res Ayurveda and Siddha 1999;XX(1-2):1-9.

34. Sharma RD, Sarkar A, Hazra DK et al.: Use of fenugreek seed powder in the management of non-insulin dependent diabetes mellitus. Nutr Res 1996a;16:1331-1339.

35. Goyal DK, Tiwari SK: Study on sadabahar (Vinca rosea Linn) in madhumeha (diabetes mellitus). J Res Ayurveda and Siddha 1999;30;XX(1-2): 93-100.

36. Chowdhary DP, Dua M, Bharti et al.: Hypoglycaemic effect of a coded formulation: Aysuh82. J Res Ayurveda and Siddha 1998;XIX(3-4): 107-115.

37. Pandey VN, Rajagopalan SS, Chowdhary DP: An effective Ayurvedic hypoglycemic formulation. J Res Ayurveda and Siddha 1995;XVI (1-2):1-14.

38. Shankar R, Singhal RK: Clinical studies of the effect of abraga (mica) chendooram in the treatment of diabetes mellitus (neerazhivu). J Res Ayurveda and Siddha 1995;XVI(3-4): 108-117.

39. Shankar R, Singhal RK: Clinical assessment of the effects of sandana (sandal) podi-a in the treatment of diabetes mellitus (neerazhiv). J Res Ayurveda and Siddha 1994;XV(3-4):89-97.

40. Sircar AR, Ahuja RC, Natu SM et al.: Hypoglycemic, hypolipidemic and general beneficial effects of an herbal mixture MA-471. Altern Ther Clin Pract 1996;3(5):26-31.

41. Maji D, Singh AK: Clinical trial of D-400, a herbomineral preparation in diabetes mellitus. J Diabetic Assoc India 1995:35(1):1-4. World Health Organization: Traditional Medicine in Asia. WHO Regional Publications, South-East-Asia Series, No. 39. WHO-Verlag (2002).

42. Yamey G. Can complementary medicine be evidence based? West J Med 2000;173:4.5

43. World Health Organization: Traditional Medicine strategy 2002-2005. WHO-Verlag, Geneva 2002.

44. Hentschel C, Kohnen R, Hauser G, Lindner M, Ernst E, Hahn EG: Entscheidung zur Komplementärmedizin: Sachorientiert oder irrational? Dt Med Wochenschr 1996;(121):1553-1560.

45. Angell $\mathrm{M}$, Kassirer J: Alternative medicine - the risks of untested and unregulated remedies. N Engl J Med 1998:339:839-841.

46. MRC. Guidelines for Good Clinical Practice in Clinical Trials. Medical Research Council, (1993).

47. SOP. Projektkoordination, Projektablauf. Arbeits gruppe Klinische Studien der Universität zu Köln Institut für Gesundheitsökonomie und Klinische Epidemiologie, Version V1.5- F1, 2000.

48. ICH (International Conference of Harmonisation of Technical Requirements for Registration of Pharmaceuticals for Human Drugs): $\mathrm{ICH}$ Consensus Guideline: Statistical Principles for Clinical Trials. 1998

49. Mehnert $H$, Standl E, Usadel K: Diabetologie in Klinik und Praxis. Georg Thieme Verlag, 5. Auflage, 2004.

50. Liebl A et al.: Kosten des Typ-2-Diabetes in Deutschland - Ergebnisse der CODE-2 ${ }^{\circledR}-$ Studie: Dtsch Med Wochenschr 2001;126:585- 589.

51. Rosenthal R: Parametric measures of effect size. In: Cooper H, Hedges LV (eds): The handbook of research synthesis. Sage, New York 1994, 231-244.

52. Hedges LV: Fixed effects models. In: Cooper $\mathrm{H}$ Hedges LV (eds.): The handbook of research synthesis. Sage, New York 1994, 231-244.

53. www.dialogforum-pluralismusindermedizin.de 2008.

\section{Disclosure Statement}

The authors declare that no financial or other conflict of interest exists in relation to the content of this article.

\section{Korrespondenzadresse:}

Dr. med. Christian H.S. Kessler Rigaerstr. 70, DE-10247 Berlin kessler.christian@gmail.com 